\title{
The challenge of managing comorbidities: a case report of primary Sjogren's syndrome in a patient with acute intermittent porphyria
}

\author{
Qing Teng ${ }^{1}$, Liyan $\mathrm{Ma}^{2}$, Yuelin Ma ${ }^{1}$, Yiran $\mathrm{Zhang}^{3}$, Ninglin $\mathrm{Kang}^{4}$, Yuanxiang $\mathrm{Hu}^{5}$, \\ Songyun Zhang,*
}

${ }^{1}$ Department of Endocrinology, The Second Hospital of Hebei Medical University, Shijiazhuang, Hebei, China;

${ }^{2}$ Department of Rheumatology, The Second Hospital of Hebei Medical University, Shijiazhuang, Hebei, China;

${ }^{3}$ School of First Clinical Medical College, Southern Medical University, Guangzhou, Guangdong, China;

${ }^{4}$ Hebei Provincial Hospital of Traditional Chinese Medicine, Shijiazhuang, Hebei, China;

${ }^{5}$ Geriatrics, First Affiliated Hospital of Hebei North University, Zhangjiakou, Hebei, China.

SUMMARY Acute intermittent porphyria (AIP) is a rare inherited metabolic disease associated with heme metabolism. Primary Sjogren's syndrome (PSS) is a common autoimmune disease. The combined presence of AIP and PSS complicates treatment. A rare case of concomitant AIP and PSS is reported in this paper. A 30-year-old woman with AIP had recurrent acute abdominal pain, nausea and vomiting, constipation, persistent chest, back, and waist pain, red urine, positivity for porphobilinogen (PBG) in urine and a pathogenic mutation of the HMBS gene. Two and a half years after she was diagnosed with AIP, she was diagnosed with PSS based on dryness of the eyes and mouth, the elevation of immunoglobulins ( $\operatorname{IgG}$ and $\operatorname{IgA}$ ) and positive results on an anti-SS-A antibody test, an anti-SS-B antibody test, Schirmer's test and a labial gland biopsy. A mutation in the HMBS gene was detected in the patient and her cousin, but the patient had more severe AIP and more severe symptoms (such as epilepsy and a limp), which may be related to the co-morbidity of PSS. According to her PSS activity score, the patient had an ESSDAI score of 9 and required systemic treatment. However, potential medications were limited by AIP, so mycophenolate mofetil was eventually added to delay the progression of the primary disease.

Keywords acute intermittent porphyria, primary Sjogren's syndrome, autoimmune disease, treatment

\section{Introduction}

Acute intermittent porphyria (AIP) is an autosomal dominant metabolic disease caused by gene mutations that results in decreasing heme synthase activity and accumulation of metabolic substrates, leading to neurological dysfunction (1). Primary Sjögren's syndrome (PSS) is a chronic inflammatory autoimmune disease that mainly affects exocrine glands such as the lacrimal and salivary glands (2) and that has a definite familial aggregation.

Many drugs cause the acute aggravation of AIP. If a patient with PSS has a score $>5$ on the EULAR Sjögren's syndrome disease activity index (ESSDAI) (indicating moderately active PSS), the patient needs to receive systemic treatment in addition to local treatment. However, the key drugs used in systemic therapy, such as methylprednisolone and cyclophosphamide, can induce the acute onset of AIP. This poses major challenges to the treatment of AIP and other comorbidities in patients with PSS.

To date, there are few reports of patients with both AIP and autoimmune diseases, and most have AIP and systemic lupus erythematosus (SLE). At present, one case of AIP and PSS has been reported in Sweden (3). Reported here is a case of AIP and PSS, and this study explores its potential pathogenesis and difficulty in terms of treatment selection.

\section{Patient and Methods}

This study was reviewed and approved by the ethics committee of the Second Hospital of Hebei Medical University, and the patient and her family provided informed consent. All clinical data of the patient were collected. The patient and her cousin underwent exon gene sequencing, and genes related to AIP and immunodeficiency disorders were analyzed. The 
ESSDAI scoring criteria were used to score the activity of PSS, and systemic treatment was required with an ESSDAI score $>5$. The safety of glucocorticoids, immunosuppressants, and biological agents was analyzed based on references from the American Porphyria Foundation (www.porphyriafoundation.com) and the European Porphyria Network (www.porphyria-europe. com).

The current case involved a 30-year-old woman who had episodic abdominal pain for six years and a dry mouth and eyes for three and a half years.

Six years ago, in the fifth week of pregnancy, the woman developed acute and severe diffuse abdominal pain, accompanied by nausea, vomiting, constipation, persistent chest, back, and lower back pain, and red urine. Examinations revealed positivity for porphobilinogen $(\mathrm{PBG})$ in urine and a mutation in the hydroxymethylbilane synthetase (HMBS) gene: c. 806C $>$ G (p.Thr269Arg) (Figure 1). The woman was diagnosed with severe AIP. In the six years after she was diagnosed, she had 17 acute episodes with two severe attacks. The first severe attack happened five years ago. The second day after undergoing a Cesarean section, the woman had severe abdominal pain, accompanied by headaches, dizziness, blurred vision, and seizures. Her blood pressure was 170/110 mmHg. A skull MRI revealed reversible encephalopathy syndrome. The second severe attack happened two years ago. The woman had severe abdominal pain after a cold, flaccid paralysis of the limbs, and muscle atrophy in the left hand. Each attack was treated with high concentration of glucose, heme arginate, an analgesic, and a beta blocker to lower blood pressure; the condition was relieved for one-three weeks.

Three and a half years ago, the woman developed a dry mouth and eyes and the sense of binocular gravel. Test results (Table 1) indicated that serum immunoglobulins (IgG and $\operatorname{IgA}$ ) were elevated, and serum anti-SS-A antibody, serum anti-SS-B antibody, Schirmer's test were positive. A labial gland biopsy revealed moderate destruction of some of the acini,

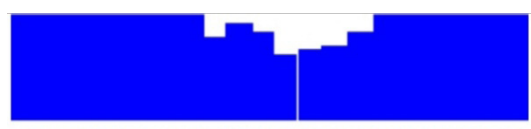

GCCGIGCATAC AGCTATGAAC

$181+193$

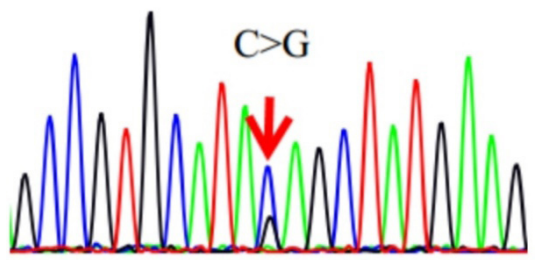

Figure 1. Detection of a mutation in the HMBS gene causing porphyria: c. $806 \mathrm{C}>\mathrm{G}$ : p.T269R. and interstitial scattering and multifocal infiltration of lymphocytes and plasma cells (grade 4) (Figure 2). Consequently, the woman was diagnosed with PSS. The woman was given artificial tears. PSS has gradually progressed over the past three and a half years. The woman developed mumps 3 times, and rampant dental caries and tooth loss gradually appeared. Serum immunoglobulin continued to rise (IgG: $16.70 \rightarrow 28.00$ g/L, IgA: $5.23 \rightarrow 6.24 \mathrm{~g} / \mathrm{L})$. Complement C3 continued to decrease $(\mathrm{C} 3: 0.91 \rightarrow 0.59 \mathrm{~g} / \mathrm{L})$.

Since the onset of AIP six years ago, routine urine testing has continued to reveal proteinuria $(+)$, and 24hour urine protein revealed proteinuria $(0.65-1.05 \mathrm{~g} /$ day) that went untreated. Five years ago, the woman was diagnosed with Hashimoto's thyroiditis (subclinical hypothyroidism), and she was given Euthyrox $50 \mathrm{ug} / \mathrm{d}$. Three years ago, intermittent purpura developed on the skin of the lower limbs after physical activity, though this was not treated.

Among the woman's family members, a cousin had similar attacks of abdominal pain, but the symptoms were mild, and there were no symptoms such as a dry mouth and dry eyes. Whole exon sequencing revealed a heterozygous missense mutation HMBS:NM_000190:exon12:c.806C > G (p.Thr269Arg) in the woman and her cousin. The results of firstgeneration sequencing indicated that the mutation was inherited from her father and not found in her mother. Analysis of the whole exon sequencing data from the woman and her cousin revealed three heterozygous missense variants related to immunodeficiency disorders: PSMB8: c. 272G>A (p.Arg91Gln), IRF8: c.105G >C (p.Met35Ile), and CR2: c.3268C $>$ A (p.Pro1090Thr) were carried by the woman but not by her cousin.

Table 1. Specific laboratory results related to dryness of the eyes and mouth

\begin{tabular}{lcc}
\hline Laboratory test & Test result & Determination \\
\hline IgA & $5.23 \mathrm{~g} / \mathrm{L}$ & $\mathrm{H}$. \\
IgG & $16.70 \mathrm{~g} / \mathrm{L}$ & $\mathrm{H}$. \\
Anti-SS-A antibody & 114 & + \\
Anti-SS-B antibody & 71 & + \\
Schirmer's test & $\mathrm{R}=2 / \mathrm{mm}, \mathrm{L}=3 / \mathrm{mm}$ & + \\
\hline
\end{tabular}

H: elevated; Positive +

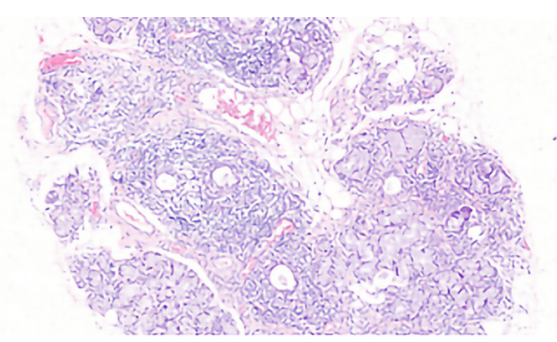

Figure 2. Microphotograph of a biopsied specimen from the patient's labial gland 


\section{Results and Discussion}

This report has described a case of AIP and PSS. On the one hand, typical clinical manifestations of AIP and a known HMBS gene mutation (c. 806C $>$ G (p.T269R)) were identified. On the other hand, there were typical clinical manifestations of PSS such as dry eyes and a dry mouth, the elevation of immunoglobulins (IgG and IgA) and the results of Schirmer's test, an anti-SS-A antibody test, an anti-SS-B antibody test, and a labial gland biopsy were all positive. A mutation in the HMBS gene was detected in the patient and her cousin, but the patient had more severe AIP, more frequent seizures, and more severe symptoms (such as epilepsy and a limp), which may be related to the co-morbidity of PSS.

SS is an autoimmune disease that primarily affects the exocrine glands and is thought to be the result of a combination of heredity and environment. Bacterial or viral infections activate the immune system, which may cause susceptible individuals with SS to develop SS (4). Accordingly, whole exon sequencing data from the patient and her cousin were analyzed. Three heterozygous missense variants related to immunodeficiency disorders were found: PSMB8: c. 272G>A (p.Arg91Gln), IRF8: c. 105G $>$ C (p.Met35Ile), and CR2: c.3268C $>$ A (p.Pro1090Thr) were carried by the patient but not by her cousin. However, whether these three variants are related to the clinical phenotype of patient needs to be studied further.

The treatment of AIP and PSS is more complex than treatment of PSS alone because drugs that may induce the acute onset of AIP must be avoided. The treatment of PSS includes symptomatic treatment and systemic treatment. According to the 2019 EULAR recommendations, patients with an ESSDAI score $>5$ require systemic treatment. The patient also had cutaneous purpura, renal damage, hypothyroidism, and other extra-glandular lesions with dry mouth and eyes. Her ESSDAI score was 9 (Table 2), so systemic treatment must be given at the same time as symptomatic treatment. Glucocorticoids are the drug of choice for the systemic treatment of PSS, and medium-acting glucocorticoids are the first option. Prednisolone and triamcinolone can be safely used in patients with AIP, but the current patient refused to take them due to concerns of being overweight and having a family history of diabetes. Immunosuppressants are a second option, with cyclophosphamide being used most often, but it can induce the acute onset of AIP and thus could not be given to the current patient. Other immunosuppressants such as mycophenolate mofetil, azathioprine, cyclosporine, and methotrexate can all be used safely (Table 3). Mycophenolate mofetil can inhibit the proliferation of activated $\mathrm{B}$ cells and reduce serum immunoglobulin levels (5), and especially in patients with PSS associated with hyperglobulinemia and glomerulonephritis (6). Cyclosporine-A mainly inhibits T cell proliferation and is nephrotoxic $(7,8)$. Azathioprine can not be used together with febuxostat (9), and has potent myelosuppressive action (10). Methotrexate is commonly used to alleviate the symptoms of arthritis (11) and skin lesions (12) in patients with PSS. In the current patient, hyperglobulinemia (-) was considered to be associated with purpura. Given renal damage and anemia in this patient, mycophenolate mofetil was

Table 2. Specific clinical manifestations indicated by a patient's disease activity score

\begin{tabular}{lll}
\hline Affected area & Level of disease activity & \multicolumn{1}{c}{ Specific manifestations } \\
\hline Glandular domain & Moderate activity $=2$ & $\begin{array}{l}\text { Major glandular swelling: Enlarged parotid glands (ultrasound of the parotid gland reveals } \\
\text { that the right parotid gland is about } 5.5 \times 2 \mathrm{~cm} \text { in size and the left parotid gland is about } 5 \times \\
1.5 \mathrm{~cm} \text { in size) }\end{array}$ \\
$\begin{array}{l}\text { Cutaneous domain } \\
\text { Renal domain }\end{array}$ & $\begin{array}{l}\text { Moderate activity }=2 \\
\text { Mederate activity }=2\end{array}$ & $\begin{array}{l}\text { Purpura of the ankle } \\
\text { Moderate renal involvement: Glomerular involvement with proteinuria (1.05 g/d) } \\
\text { Biological domain }\end{array}$ \\
\hline
\end{tabular}

Total ESSDAI score: 9.

Table 3. Safety of glucocorticoids, immunosuppressants, and biological agents commonly used for PSS in patients with AIP

\begin{tabular}{|c|c|c|c|}
\hline Items & Glucocorticoid & Immunosuppressant & Biological agent \\
\hline NP/PNP & $\begin{array}{l}\text { Prednisolone (Medium-acting) } \\
\text { Triamcinolone (Medium-acting) } \\
\text { Betamethasone (Long-acting) }\end{array}$ & $\begin{array}{l}\text { Mycophenolate mofetil } \\
\text { Azathioprine } \\
\text { Methotrexate } \\
\text { cyclosporine-A }\end{array}$ & Rituximab \\
\hline PSP & $\begin{array}{l}\text { Hydrocortisone (Short-acting) } \\
\text { Cortisone (Short-acting) } \\
\text { Prednisone (Medium-acting) } \\
\text { Methylprednisolone (Medium-acting) } \\
\text { Dexamethasone (Long-acting) }\end{array}$ & Cyclophosphamide & \\
\hline
\end{tabular}

NP, not porphyrinogenic; PNP, probably not porphyrinogenic; PSP, possibly porphyrinogenic. 
the most suitable. A biological agent is a third option. Rituximab can be safely used in patients with PSS and severe hemocytopenia or lymphoma (13), but this drug was not required in the current patient. Since the patient was given artificial tears to relieve dry eyes, she was given mycophenolate mofetil to delay the progression of the primary disease.

\section{References}

1. Benassi F, Righi E, Cimato P, Parravicini R. Cardiac surgery in patients with acute intermittent porphyria. J Card Surg. 2012; 27:331-4.

2. Ramos-Casals M, Brito-Zerón P, Sisó-Almirall A, Bosch X. Primary Sjogren syndrome. BMJ. 2012; 344:3821.

3. Floderus Y, Shoolingin-Jordan PM, Harper P. Acute intermittent porphyria in Sweden. Molecular, functional and clinical consequences of some new mutations found in the porphobilinogen deaminase gene. Clin Genet. 2002; 62:288-697.

4. Shen L, He J, Kramer JM, Bunya VY. Sjögren's Syndrome: Animal Models, Etiology, Pathogenesis, Clinical Subtypes, and Diagnosis. J Immunol Res. 2019; 2019:8101503.

5. Allison AC, Eugui EM. Mycophenolate mofetil and its mechanisms of action. Immunopharmacology. 2000; 47:85-118

6. Bertsias GK, Tektonidou M, Amoura Z, et al. Joint European League Against Rheumatism and European Renal Association-European Dialysis and Transplant Association (EULAR/ERA-EDTA) recommendations for the management of adult and paediatric lupus nephritis. Ann Rheum Dis. 2012; 71:1771-1782.

7. Lai Q, Luo Z, Wu C, Lai S, Wei H, Li T, Wang Q, Yu Y. Attenuation of cyclosporine A induced nephrotoxicity by schisandrin B through suppression of oxidative stress, apoptosis and autophagy. Int Immunopharmacol. 2017; 52:15-23.

8. Caires A, Fernandes GS, Leme AM, Castino B, Pessoa
EA, Fernandes SM, Fonseca CD, Vattimo MF, Schor N, Borges FT. Endothelin-1 receptor antagonists protect the kidney against the nephrotoxicity induced by cyclosporine-A in normotensive and hypertensive rats. Braz J Med Biol Res. 2017; 51:6373.

9. Logan JK, Wickramaratne Senarath Yapa S, Harinstein L, Saluja B, Muñoz M, Sahajwalla C, Neuner R, Seymour S. Drug interaction between febuxostat and thiopurine antimetabolites: a review of the FDA Adverse Event Reporting System and medical literature. Pharmacotherapy. 2020; 40:125-132.

10. Stoenoiu MS, Aydin S, Tektonidou M, et al. Repeat kidney biopsies fail to detect differences between azathioprine and mycophenolate mofetil maintenance therapy for lupus nephritis: Data from the MAINTAIN Nephritis Trial. Nephrol Dial Transplant. 2012; 27:19241930.

11. Mirouse A, Seror R, Vicaut E, et al. Arthritis in primary Sjögren's syndrome: characteristics, outcome and treatment from French multicenter retrospective study. Autoimmun Rev. 2019; 18:9-14.

12. Argyropoulou OD, Tzioufas AG. Common and rare forms of vasculitis associated with Sjögren's syndrome. Curr Opin Rheumatol. 2020; 32:21-28.

13. Leanna MW, Daniel GA. A patient with primary Sjogren's syndrome, cystic lung disease, and MALT lymphoma treated successfully with rituximab: A case-based review. Clin Rheumatol. 2020; 39:1357-136.

Received June 2, 2020; Revised July 18, 2020; Accepted July 29, 2020.

*Address correspondence to:

Songyun Zhang, Department of Endocrinology, The Second Hospital of Hebei Medical University, Shijiazhuang 050000, Hebei, China.

E-mail: zsy2020@hebmu.edu.cn

Released online in J-STAGE as advance publication August 6, 2020. 